\title{
BMJ Open Supporting surgeons in patient-centred complex decision-making: a qualitative analysis of the impact of a perioperative physician clinic
}

\author{
Clarabelle T Pham (D) , ${ }^{1,2}$ Catherine L Gibb, ${ }^{3,4}$ Robert A Fitridge, ${ }^{4}$ Jon Karnon, ${ }^{1}$ \\ Elizabeth Hoon ${ }^{5}$
}

To cite: Pham CT, Gibb CL, Fitridge RA, et al. Supporting surgeons in patient-centred complex decision-making: a qualitative analysis of the impact of a perioperative physician clinic. BMJ Open 2019;9:e033277. doi:10.1136/ bmjopen-2019-033277

- Prepublication history for this paper is available online. To view these files, please visit the journal online (http://dx.doi. org/10.1136/bmjopen-2019033277).

Received 29 July 2019 Revised 21 November 2019 Accepted 29 November 2019

Check for updates

(C) Author(s) (or their employer(s)) 2019. Re-use permitted under CC BY-NC. No commercial re-use. See rights and permissions. Published by BMJ.

For numbered affiliations see end of article.

Correspondence to Dr Clarabelle T Pham; clara.pham@flinders.edu.au

\section{ABSTRACT}

Objective Patients with comorbidities can be referred to a physician-led high-risk clinic for medical optimisation prior to elective surgery at the discretion of the surgical consultant, but the factors that influence this referral are not well understood. The aims of this study were to understand the factors that influence a surgeon's decision to refer a patient to the clinic, and how the clinic impacts on the management of complex patients.

Design Qualitative study using theoretical thematic analysis to analyse transcribed semi-structured interviews. Setting Interviews were held in either the surgical consultant's private office or a quiet office/room in the hospital ward.

Participants Seven surgical consultants who were eligible to refer patients to the clinic.

Results When discussing the factors that influence a referral to the clinic, all participants initially described the optimisation of comorbidities and would then discuss with examples the challenges with managing complex patients and communicating the risks involved with having surgery. When discussing the role of the clinic, two related subthemes were dominant and focused on the management of risk in complex patients. The participants valued the involvement of the clinic in the decision-making and communication of risks to the patient.

Conclusions The integration of the high-risk clinic in this study appears to offer additional value in supporting the decision-making process for the surgical team and patient beyond the clinical outcomes. The factors that influence a surgeon's decision to refer a patient to the clinic appear to be driven by the aim to manage the uncertainty and risk to the patient regarding surgery and it was seen as a strategy for managing difficult and complex cases.

\section{INTRODUCTION}

The decision of whether to operate on a patient can be complex. For patients who are young and fit with no other medical comorbidities, the decision to proceed with surgery is relatively straight-forward. In contrast, the decision to operate on a patient who is frail and/or has multiple medical comorbidities can be challenging, and could potentially
Strengths and limitations of this study

- This is the first empirical study to explore surgeons perspectives about the value of a physician-led high-risk clinic for the management of complex surgical patients.

- Data saturation of themes was achieved for the surgeons who frequently referred patients to the clinic due to the in-depth data obtained on a specific interview topic.

- A small sample from a difficult to access population limits the generalisability of the findings; however, this approach generates in-depth insights which may inform the future development of outcome measures for such services.

- The lack of participation by surgeons choosing not to use the clinic limits the ability to draw conclusions on aspects of the clinic that may be considered low value.

- Additional perspectives from interviews with other stakeholder groups would provide further insights into the aspects of care of complex patients.

involve a large degree of uncertainty due to the increased risk of morbidity and mortality during the perioperative period. ${ }^{12}$

A set of potentially modifiable comorbidities have been identified to be associated with increased length of hospital stay and postoperative complications. ${ }^{3}$ Patients with poorly controlled modifiable comorbidities can be referred for medical optimisation prior to elective surgery at the discretion of the surgical consultant, but the factors that influence this referral are not well understood. ${ }^{4}$ Further, there has been minimal detail published on the actual services provided as part of the medical optimisation of patients and its design and implementation has been heterogeneous to date. ${ }^{4}$

The number of patients with multiple comorbidities increases with an ageing population. Outpatient clinics, such as the 
Perioperative High Risk Clinic at the Royal Adelaide Hospital in South Australia, have been established to comanage complex patients by targeting, managing and optimising their modifiable medical comorbidities. The current model of care for this clinic involves the ad hoc referral of patients who have been identified by either the surgeon or anaesthetist as having the potential to benefit from preoperative assessment and management. The clinic is funded by local government and comprises two part-time physicians, one clinical practice nurse consultant and one administration officer. On average, 16 new patients are assessed and eight patients are reviewed per week in addition to the comanagement of assessed patients who have been admitted to hospital for surgery. A recent evaluation of this clinic $^{5}$ was found to reduce the frequency of unnecessary admissions for and cancellations of surgery but there was significant uncertainty around the effect of the clinic on clinical outcomes and costs. The evaluation included a range of measured factors, such as patient demographics, surgery-related factors and selected medical comorbidities, that could bias or distort the association between the clinic and patient outcomes. However, the evaluation findings suggested that there may be additional unmeasured factors that contribute to patient complexity, ${ }^{3}$ thereby influencing referral to the clinic and the potential underestimation of the effect of the clinic on patient outcomes.

This explanatory study was designed to provide an in-depth examination of the surgeons' perceptions of referral practices to the clinic and thereby illuminate the findings of the clinic evaluation. Factors related to the referral of patients for medical optimisation identified by the surgeons but not captured by the available data would indicate the potential presence of unmeasured confounding in the clinic evaluation. Additionally, the surgeons' perceptions of the clinic would inform whether the value of the clinic was appropriately captured in the outcomes used in the clinic evaluation. The aims of this study were to understand the factors that influence a surgeon's decision to refer a patient to the clinic, and how the clinic impacts on the management of complex patients.

\section{Methods}

\section{Research design}

This explanatory sequential study design ${ }^{6}$ was part of a larger mixed methods study evaluating the assessment and management of high-risk patients provided by an outpatient Perioperative High Risk Clinic ('the clinic'). The qualitative data collected was used to explain and build on the findings of the quantitative evaluation of the services provided by the clinic.

Taking a realist epistemological approach, theoretical thematic analysis was used to analyse the collected data. ${ }^{7}$ There was a focus on themes to help explain the complexity of the patient population referred to the clinic and provide insight into the context, subjective attitudes and behaviours that govern the surgeons' decision making processes in assessing and managing complex surgical patients.

\section{Setting and participants}

Semi-structured interviews were conducted in person with surgical consultants from five specialties at the Royal Adelaide Hospital and The Queen Elizabeth Hospital, Adelaide, South Australia. Participants were recruited with an invitation letter via post or electronic mail and followed up with a telephone call. Sampling was purposive and included surgical consultants with varying levels of experience from several specialties who were eligible to refer patients to the clinic and could provide in-depth knowledge on the decision-making process for referral and management of complex patients. The aim was to achieve data saturation given the specific interview topic.

\section{Data collection}

The interviews were conducted by one of the authors (CTP), a researcher with no clinical relationship with patients or staff of the clinic but who had recently completed the quantitative evaluation of the clinic, between March and July 2014. There was a potential for bias given the researcher's working relationship with the clinic but the evaluation of the clinic (both quantitative and qualitative) was funded by an independent government-funded agency and there was no dependent working relationship between the researcher and the clinician beyond the study. One-to-one interviews were held in either the surgical consultant's private office or a quiet office/room in the hospital ward. Prior to being interviewed, participants received an information sheet and completed a consent form.

The study team developed an interview guide and compiled interview and probing questions focusing on the reasons for and expectations of referral to the clinic, and the level of involvement of the clinic in the surgical decisionmaking process and the delivery of clinical care to patients. The questions and prompts in the guide were developed by the project investigators (academic researchers, the clinic physician and a surgeon) and piloted on selected surgeons to test the appropriateness and flow of questions.

All interviews were audio recorded and transcribed by either the interviewer, or a professional transcription service and reviewed by the interviewer to ensure accuracy.

\section{Data analysis}

A theoretical approach was used to develop themes from the data. Given the specific questions, explanatory purpose and area of interest, themes were identified at a semantic level focusing on the explicit, stated meaning of the data. ${ }^{7}$ CTP initially coded all transcripts and $\mathrm{EH}$ reviewed and coded selected transcripts. CTP and EH examined the consistency of the coded text and explored and refined the emerging themes. Codes were initially organised into broader thematic groups and subthemes, which were then reviewed and discussed within the research team to check the relevance of themes with clinicians and compare 
findings to contemporary literature about clinical decisionmaking and managing risk. All authors contributed to the final selection of themes and their interpretation in relation to the overall research questions.

NVivo 10 for Windows (QSR International) was used to transcribe, code and manage data.

Written informed consent was obtained from each participant, including permission to audio-record the interviews and use anonymised quotes.

\section{Patient and public involvement}

Patients were not involved in the design, recruitment and conduct of the study. Participants will be notified of published study findings.

\section{Findings}

\section{General characteristics}

Of the 27 invitations sent, seven surgical consultants agreed to participate (one of whom referred patients infrequently to the clinic). The reasons for non-participation were not known. Three females and 17 males from the following surgical specialties did not respond to the invitation to participate: anaesthetics; ear, nose and throat surgery; upper gastrointestinal surgery; orthopaedic surgery; urology; colorectal surgery; vascular surgery and breast cancer surgery. The duration of the interviews ranged from 10 to $34 \mathrm{~min}$ (mean $23 \mathrm{~min}$ ). Interviewees were predominantly male, with surgical experience ranging from 11 to 31 years, indicating that all had quite extensive experience on which to draw on in the interview. The surgical specialties included vascular surgery; colorectal surgery; orthopaedic surgery; ear, nose and throat surgery; and general and hepatobiliary surgery. For participants who frequently referred patients to the clinic, data saturation of themes was achieved.

Two main themes were readily identified and explored in this analysis: factors that influence a surgeon's decision to refer a patient to the clinic, and the role of the clinic in assessing and managing complex patients.

\section{Theme 1: factors that influence a surgeon's decision to refer a patient to the clinic}

In discussing how they assessed and determined whether a patient should be referred to the clinic, participants generally identified at least two of the following factors. All participants initially described the optimisation of comorbidities and would then elaborate with discussion of exemplar patient cases to demonstrate the challenges with managing complex patients and communicating the risks involved with having surgery.

\section{Optimisation of comorbidities}

All participants, including the participant who referred patients infrequently, mentioned the optimisation of comorbidities as one of their main expectations when referring a complex patient to the clinic and felt that it complemented the care they provided.
I see the role of it as identification of patients who have a higher risk of morbidity and mortality related to the [...] surgical treatment of their disease, which are largely cancer diseases, and after identification of their risk, then to optimise their care to reduce that risk to the lowest possible level for surgery. (Participant 3)

And

... their medical issues are examined or delineated and any further investigation that might be warranted is undertaken, medications may be changed $[\ldots]$ and in the process, though I see the optimisation probably more as the role, it gives the patient further insight into what sort of medical risks are involved and potential implications. So it adds to the informed decision making process for consent. (Participant 2)

\section{And}

I expect probably a more thorough assessment from a medical disease point of view, an optimisation of their conditions whether it's an adjustment of their inhalers or their cardiac medications or whatever. Some more streamlined advice on what they should be doing with their medications in the perioperative period. (Participant 5)

The assessment and review of current medical conditions and medication management in complex patients appears to be the main reason for referral to the clinic. The focus on the impact of such medical conditions on the surgical risk was seen to add to the decision making process and to improve patient informed consent.

\section{Management of factors other than comorbidities}

Beyond the checklist of potentially modifiable comorbidities that can be optimised by the clinic, there appeared to be unmeasured factors that contributed to the surgeon's decision to refer, such as a patient's physiological reserve and resilience. When discussing the decision to refer a complex patient to the clinic, all participants referred to clinical experience and judgement in discussing how they assessed increased risks and uncertainty during and after surgery.

... we don't use an algorithm to decide, it's just an experiential thing and so I think of patients as oh my goodness they're going to be quite difficult to look after afterwards, I think we should get some help beforehand. You just look at them and go you're going to break if I touch you. It's a resilience thing in a way. We talk about psychology but it's a physical resilience to see how much reserve do you think they're going to have. Therefore if it's very low then you need to make sure that everything goes absolutely perfectly otherwise it's going to be a disaster. (Participant 4)

And

I think it's identifying patients that have a low reserve so while they might not have identifiable medical problems you recognise that they are patients that 
potentially don't do well after the operation and I suppose that we accept that any major surgery takes someone down a level of functioning so if someone's only just functioning that's going to be significant. I suppose with fragility, we look at how they cope with everyday function as a sort of measure of how robust they are. (Participant 2)

And

So just going to the waiting room, walking them in, sitting them down, getting them up onto the bed. I mean if it's hard work to get them up onto the bed, you need to crow bar them onto the bed or whatever, a bit of a red flag will go up. How far they can walk or activities of daily living, if it's quite challenging. (Participant 1)

\section{And}

You get a pretty good idea of frailty in the first 90 s when they walk into the room, really. It's just sort of clinical acumen, I suppose. That probably sounds a bit snobby. But you can just tell-if you sat on a bus and looked at the old people on the bus, you'd think, well, she looks pretty good for 70 and the man looks like he needs help to sit down or to stand up. (Participant 6)

As the previous quotes highlight, there was variance in how participants discussed their use of clinical experience and judgement; however, these assessments commonly focused on physical dimensions of reserve and fragility. One participant described the combination of physical and cognitive characteristics that could also prompt a referral to the clinic, suggesting informal checks for indicators of frailty.

Strength of handshake, mobility, cognitive ability at answering questions, focusing properly, how they interact with their family. All those things I think all add up to bizarrely an increased operative risk which we somehow try and translate into needing to see a specialist about that beforehand. (Participant 4)

There appears to be a stronger focus on the patient's physical characteristics by observing their ability to perform usual activities than the cognitive and psychosocial functioning, but difficulties with both the physical and cognitive aspects was seen to increase the operative risk. Despite participants commonly describing the informal checks for some of the indicators of frailty, none identified the use of a quantitative measure.

\section{Strategy for managing difficult and complex cases}

Given the complexity of the patient and the uncertainty regarding surgical risk, some participants viewed the clinic as a means to provide an independent, second opinion on whether the patient should proceed with surgery.

But as I say, having that second set of eyes or third-party assessment $[\ldots]$ because it's very difficult where a patient's come along with an expectation that they need something done. They generally expect that we're going to see them and say, right, well, yes, away we go. If you try to tell them that maybe, really, it's not the best thing to do, I think it's very useful to have a third party who's clearly disconnected from the surgical clinic and say, look, you're actually not-do you really want to do this? Do you realise this is a bit risky? (Participant 6)

And

I mean probably the most common reason I use the clinic is for the patients to understand the risk involved in surgery. So patients I don't particularly want to operate on, for me that's the ideal situation. The patient says why can't you do this $[\ldots]$ why is it risky? So they go and see [the clinic physician] and then [the clinic physician] tells them. It helps put the thing in perspective for them. (Participant 7)

These examples highlight how the referral for a second opinion not only provides an additional clinical assessment but also a strategy to manage the expectations of patients, particularly in communicating the risks with surgery.

One of the participants mentioned the challenges with cancellations to the surgery schedule but it did not appear to be a key factor in the decision to refer a patient.

\section{Holistic assessment and care of the patient}

Referral to the clinic is currently ad hoc and largely at the discretion of the surgeon. Prior to the establishment of the clinic, surgeons would refer patients to subspecialists, such as the cardiologist and nephrologist. One participant in this study continued with this practice and very rarely referred patients to the clinic.

... I've been around for a long time and used to operating on high risk patients without having someone review them, so we've got a pretty good understanding of what risks are involved. A pretty good understanding of who needs to have something done [...] Often if patients need an operation then there's not much that the high risk clinic can do. I mean if things are-medical conditions are not treated optimally then that's a role for the high risk clinic. I don't really need a high risk clinic to tell me this patient might have an infarct, they might have a stroke when I'm doing an operation, to prevent it [...] They've by and large seen other people. Other people manage those things, if everything seems to be controlled. If you can't correct something then they either have to have the operation or they don't. (Participant 7)

This participant distinguished the surgical from the medical and perceived the clinic to have a role in reviewing medical conditions that were not well managed, but this participant did not feel they required this additional clinic review to supplement their surgical practice. In contrast, those who referred patients to the clinic frequently viewed it as a means to provide a snapshot of the overall health of a patient with multiple medical 
comorbidities, as opposed to stand-alone reports from other subspecialists.

... these patients, they've usually been cigarette smokers or possibly alcoholics as well, and so related to their lifestyle choices they often have comorbidity issues related to that, such as issues of cardiac/pulmonary disease sometimes renal disease, diabetes and when people have more than one area of problem, this is when the problem occurs. The time to get patients in to see each individual clinic, getting a report from each clinic, and trying to coalesce the importance of each of these comorbidities into one single answer is really what the problem was. So we see the benefit of the high risk clinic as the coalescence of the risks of all of their comorbidity and giving us an overall idea about the patient. (Participant 3)

And

... it's also a bit more holistic so rather than looking at each individual medical problem that a specialist might see someone for, we've got someone who integrates the whole thing, which is very beneficial. (Participant 2)

And

... the high-risk clinic is able to access the various bits of information or plug these people into clinics to get extra bits done that we can't. Before this clinic started, if I saw someone who had a cardiac history, I'd say to them, well, we can do your operation but you have to go and see the cardiologist first. So then they'd go and they'd sit in the queue for the cardiac clinic, which might be forever. Or they'd see the registrar who'd never seen them before and things—and it'd just-so this really pulls together all the bits. (Participant 6 )

And

I think a lot of specialists just compartmentalise - I'll worry about the heart and the respiratory guy will just worry about the lungs and you'll see that quite a lot that noone puts it all together. So I think that's important-. It's an integration of all the different disease processes going on.

(Participant 1)

The holistic assessment of the patient provided by the clinic was described by many participants as a consolidation of all the relevant patient factors that support their clinical decision making processes and streamlines patient care, a service not provided elsewhere. It was thought that the medically focused role of the clinic physician complemented their role allowing them to focus on the surgery. However, this perceived value was not held by all participants, which may explain the continued ad hoc nature of patient referrals to the clinic.

Theme 2: the role of the clinic in assessing and managing complex patients

When discussing the role of the clinic, two related subthemes were dominant and focused on the management of risk in complex patients. The participants emphasised the risks for both patients and surgeons and that the involvement of the clinic in the decision-making process and communication of risks, particularly in predicting how the patient would cope post-surgery, was valued by surgeons.

\section{Informing and assuring the patient}

The clinic provided additional detail on the risks to the patient and the potential implications of surgery, and was perceived to be beneficial to patients who were concerned and hesitant about having surgery.

It's useful for us but I think it's also useful for the patient, knowing what's expected, how they're going to be managed postoperatively to try and minimise their risk of pneumonia, etc. [...] I see the optimisation probably more as the role, it gives the patient further insight into what sort of medical risks are involved and potential implications. So it adds to the informed decision making process for consent. (Participant 2)

It also appeared to be beneficial for patients who were adamant about undergoing surgery despite having a high risk of morbidity and mortality.

I think for those that need it, a more clear indication of risk that they're putting themselves at. We can only tell them so much about the complications of hip replacement, but if they've got other illnesses their risk of mortality and other perioperative morbidities I think it would be important to be assessed as well. (Participant 5)

The above example also describes the separation of roles with the surgeon discussing the potential complications of the surgical procedure and the clinic physician providing supplementary information on the potential complications as a result of the patient's medical conditions. This reinforces the focus on adding to the decision making process and informed patient consent and appeared to provide a form of assurance for the patient.

Now the way that I'd probably view it most importantly though is that from the patient's point of view, they are often worried and scared, they view their outcome as being uncertain $[\ldots]$ they're worried that they're going to die, not from the cancer but from the impact of having an operation [...] If [the clinic physician] says to the patient, 'I think that you are fit to go through this type of procedure', they feel confident about that. Patients often have a traumatic time psychologically, going through management of cancer, the diagnosis and treatment of it, but if everybody that's around them works in that team, they feel happier with that. (Participant 3)

\section{Assuring the surgeon}

Descriptions of the relationship between the surgical team and the clinic revealed that there was ongoing communication and involvement in the management of the patient between the two parties throughout the surgical period, and not just a one-off consultation. This 
appeared to provide a form of assurance for the surgeon and the surgical team.

I think communication between the high risk clinic staff and the referring surgeon is important because there can be discussion about how important a procedure is relatively to treating the patient conservatively [...] Often [the clinic physician will] ring me up and say, 'can we wait 3 or 4 weeks, I'll get these tests done and I'll start a meeting, $[\ldots]$ is it safe to do that?' Usually there's quite good communication. (Participant 1 )

\section{And}

I find it useful when [the clinic physician] rings me to say that this is a real issue or there's a patient that is a bit outside the norm or their risk is going to be unacceptably high that that personal contact has been useful because it makes sure that is recognised and acted upon. (Participant 2)

\section{And}

I think it's useful for everyone who's involved because if there's some concern on the day that they come into theatre, the anaesthetist will say, well, yes, he's been to the high-risk clinic, he's a bit crumbly, but they seem to have sorted it. I think it gives the anaesthetist a bit more reassurance as well. (Participant 6)

For some specialties, the clinic physician was seen as a member of the surgical team and one who had expertise to medically manage the patient in preparation for surgery.

[the clinic physician is] an embedded part of our unit, which is critical. [...] it's not just a referral to a clinic but it's an embedding of a physician within the unit who gives us expert opinion on those areas. [...] [the clinic physician] probably has more expertise than any other physician in the hospital who doesn't see our patients regularly. [the clinic physician is] a specialist physician for surgeons. [the clinic physician] knows more about surgery than most physicians. (Participant 3)

And

[the clinic physician] comes to our radiology meetings so [the clinic physician is] part of the unit [...] often if I talk to [the clinic physician] I say, 'well ideally we should do an open because the anatomy's not favourable but if they're really high risk we may change to doing endoluminal even though the long-term results aren't as good'. I think it's useful to have that discussion in complicated cases or cases which are certainly not that straight forward. And it goes both ways, us letting [the clinic physician] know what the scoop is and [the clinic physician] maybe saying, 'you need this, this and this and you can have an open' (Participant 1)

The clinic was perceived to be beneficial for both the patient and surgeon and helped in determining the most appropriate treatment pathway, especially for complex cases.

\section{Supporting postoperative management}

Management of the patient also extended to the postoperative period, with the clinic physician providing recommendations for postoperative care of the patient and the perioperative involvement of clinic nurses (as part of the clinic team) ensuring consistency of care and appropriate postoperative follow-up.

[the clinic's] role to me now is optimising patient care and time of surgery with preoperative care but also perioperative care with the perioperative physician team. So I think it's only fair to get people involved in looking after people postoperatively only if they've had a role in preoperatively. (Participant 4)

The postoperative management provided by the clinic physician and team not only focuses on the potential medical complications after surgery but extends to managing the patient's expectations regarding care and assistance after they are discharged from hospital, as described in the following quote:

I guess there's two things, one is if there is an intervention required for example they need a coronary stent or something like that, then that's kind of a nobrainer, but if there are issues that maybe require convalescence afterwards, high risk of confusion afterwards, those sorts of things that's useful advice but you have to take into account that that doesn't usually affect necessarily decision making for the procedure. But there are a reasonable number of patients that are just coping at home and if they do have a major procedure then they'll probably never get home again and that's important because $a$ lot of patients want their independence and have no concept that a major intervention may end up with them going to a care facility on a permanent basis not just for some convalescence. (Participant 1)

All participants acknowledged the importance of the clinic's involvement in the postoperative management of their patients. However, the clinic physician's recommendations for postoperative care were viewed to be for the benefit of the medical and nursing staff who were involved with the day-to-day care of the patient in the postoperative period.

\section{DISCUSSION}

This explanatory study aimed to assess the validity of findings from a recent evaluation of an outpatient Perioperative High Risk Clinic that optimises surgical patients with a high risk of morbidity and mortality by targeting and managing their modifiable medical comorbidities. ${ }^{5}$ This study focused on identifying factors that influence a surgeon's decision to refer a patient to the clinic and the impact of the clinic on the management of complex patients. 


\section{Validity of the evaluation findings}

The recent evaluation of the clinic ${ }^{5}$ controlled for potential confounding by using a range of patient-level factors that could potentially influence whether a patient was referred to the clinic. Factors were limited by the routinely collected data available and included patient demographics, surgery-related factors, patient's physical function (using proxy measures such as the ability to walk around the house and upstairs) and the modifiable medical conditions targeted for optimisation by the clinic. The results varied depending on the method used to control for differences in observed potential confounders suggesting that the inclusion of other unmeasured confounding factors would improve the characterisation of the complexity of patients referred to the clinic.

Findings from this study suggest a high likelihood of unmeasured confounding in the quantitative evaluation of the clinic and additional relevant outcomes that could be collected to describe the perceived value of the clinic. The presence of potentially modifiable comorbidities that increase the risk of morbidity and mortality in patients was confirmed to be the initial indicator for a clinic referral for optimisation, thereby supporting the covariables used in the quantitative evaluation to control for confounding. However, unmeasured factors such as the surgeon's subjective clinical assessment of the patient and the informal use of frailty indicators also appeared to influence whether a patient was deemed to be complex and requiring additional management and treatment. This is consistent with previous studies ${ }^{89}$ reporting that associations between preoperative consultation and the presence of medical comorbidities only explained a small proportion of the substantial variation in referral patterns for consultation. This small study provides further insight into other possible sources of variation in such referral patterns.

Participants within this study described consideration of the patient's physiological reserve and resilience, indicated mainly by physical characteristics such as the ability to walk from the waiting room to the consult room, strength of handshake, and general physical function, as contributing factors to their decision to refer a patient to the clinic. Cognitive and psychosocial functioning were considered but were not the main focus for participants. The clinic includes a mini-mental state examination for cognitive impairment and the likelihood of postoperative delirium as part of the medical assessment, which appears to complement this aspect of patient care for the participants. Frailty and quality of life measures, not routinely collected in clinical data, could potentially enhance the quantitative evaluation by improving the characterisation of clinic patients. However, other aspects of the surgeon's subjective assessment, such as clinical judgement, are difficult to quantify and their influence on the reasons and thresholds for referral warrant consideration. Given the current ad hoc referral of patients to the clinic, it is likely that clinical judgement could be a contributor to the variations in the results from the quantitative evaluation.
Clinical decision making is a complex process, ${ }^{10}$ dependent on the surgeon's clinical judgement in conjunction with evidence-based practice to provide quality patient care ${ }^{1112}$ and the patient's anatomy, physiology and wellbeing. ${ }^{13}$ Clinical judgement has been described to involve critical thinking, reflective practice, problem solving, judgement, ethical values and professional accountability. ${ }^{14}$ With the accumulation of data from various clinical experiences, this judgement could be interpreted as a cognitive process involving pattern recognition that draws on the interpretation of clinical evidence within the experience, understanding and prior knowledge of the practitioner ${ }^{15}$ and differentiates the novice from the expert practitioner. ${ }^{16}$ Unfortunately, there are no reliable and validated measures of clinical judgement ${ }^{10}$ but further research should acknowledge that variation exists and focus on how thought processes of the expert practitioner, such as the types of questions asked of the patient and the diagnostic thinking process, determine the most appropriate treatment pathway. ${ }^{17}$ This could inform and improve the shared decision making process and provision of patient-centred care.

\section{Perceived value of the clinic}

Participants described additional value in the form of an overall assessment of the current health status of a patient, and the benefits of an integrated approach regarding information and assurances to both the patient and the surgeon beyond the clinical outcomes measured in the quantitative evaluation.

Clinical outcome data, such as surgical cancellations, length of hospital stay, postoperative complications, mortality and quality of life, are important to demonstrate the cost-effectiveness of preoperative medical consultations but the additional perceived value of the clinic may not be adequately captured in such clinical outcome measures. ${ }^{5}$ The reduction in uncertainty and the discussions regarding risk and expectations postsurgery, elements of value evident in this study, warrant consideration when assessing the value of healthcare interventions such as the high-risk clinic. However, how best to measure and include such elements of value and other patient-reported outcomes into cost-effectiveness analysis remains a subject of debate. ${ }^{18}$

The surgeon and clinic physician relationship has also not been well described in previous studies, ${ }^{4}$ making it difficult to determine whether the clinic physician provided a stand-alone consultation with no postoperative patient follow-up or an integrated approach to patient care between the surgeon and clinic physician. The clinic physician in this study was considered a member of surgical teams with expertise on the medical management of patients in preparation for surgery. The physician focuses on the optimisation of the patient's pre-existing medical comorbidities given the potential anaesthetic and surgical burden for the patient, which complements the skillset of the anaesthetic team focused on the provision of safe anaesthesia and specific perioperative 
management. Referred patients received preoperative assessment and management in an outpatient setting (ie, up to 12 months prior to hospital admission for surgery) and, once admitted to hospital for surgery, were comanaged by the high-risk clinic physician and nurse up until discharge from hospital. When appropriate and at times at the surgeon's request, the clinic physician may also discuss non-surgical options such as medical management and palliative care services with patients, and completion of an Advanced Care Directive (legal arrangements for the patient's future health, end of life, preferred living arrangements and other personal matters). There was ongoing communication and active involvement in the management of the patient between the surgeon and the clinic physician throughout the surgical period. Participants valued the role of the clinic in providing additional information and the opportunity for further discussion of the risks and potential implications with surgery to help with the management of patient expectations after surgery. Good communication has the potential to improve overall patient care coordination and the patient experience. ${ }^{19}$

\section{Strengths and limitations}

In this study, we have explored surgeons' perspectives of the clinic in detail as it is the surgeon who authorises the referral of a patient for preoperative assessment and management. The small sample of participants limits the generalisability of the findings to other clinics providing a similar service. The lack of participation by clinicians choosing not to use the clinic is also a limit to the study. We invited 27 surgeons and anaesthetists to participate with the understanding that this was a difficult population to access given the demands of their profession. However, given the specific interview topic, the surgeons who did make time to participate were able to provide in-depth data on the management of complex patients. Consequently, data saturation of themes was achieved for the surgeons who frequently referred patients to the clinic. Unfortunately, only one surgeon who referred patients to the clinic infrequently agreed to participate. Additional time and resources may have allowed for the collection of additional data and themes for surgeons who referred patients infrequently but this was beyond the scope and timeframe of this study. The assurances provided to both the patient and the surgeon through the assessment and management of risk and uncertainty regarding surgery should be explored through further qualitative interviews or validated shared decision-making questionnaires. Additional perspectives from the patients, the clinic team and other medical professionals collaborating with the clinic would also provide further insight into the aspects of care that provide additional value.

Decisions to invest in services such as those provided by the clinic are driven by their impact on the costs and health benefits. The development, validation and evaluation of a checklist, based on the key factors identified through qualitative and quantitative evaluations, could guide the assessment and selection of patients who would benefit most from such services and enable a robust evaluation of the clinic. Ensuring the optimal use of such services, as opposed to the ad hoc nature of the current referral process, would improve the effectiveness of existing clinics and inform the implementation of clinics at other hospitals.

\section{CONCLUSIONS}

The integration of the preoperative medical consultative service provided by the clinic in this study appears to offer additional value in supporting the surgical decision-making process for the surgical team and patient beyond the clinical outcomes. The factors that influence a surgeon's decision to refer a patient to the clinic appear to be driven by the aim to manage the uncertainty and risk to the patient regarding surgery and it was seen as a strategy for managing difficult and complex cases. The current ad hoc nature of determining which patient to refer to the clinic, as described by the participants in this study, may explain the variations in the clinical outcome measures from the quantitative evaluation, and suggest a high likelihood of unmeasured confounding, with frailty and quality of life measures not captured in current routinely collected hospital data. Further research is required to explore the perspectives of the patient, the clinic team and other medical professionals collaborating with the clinic and guide the optimal use of the preoperative medical consultative service. This will inform improvements to the design, implementation and evaluation of preoperative medical assessment and management.

\section{Author affiliations}

${ }^{1}$ College of Medicine and Public Health, Flinders University, Adelaide, South Australia, Australia

${ }^{2}$ School of Public Health, Faculty of Health and Medical Sciences, The University of Adelaide, Adelaide, South Australia, Australia

${ }^{3}$ Perioperative High Risk Clinic, Royal Adelaide Hospital, Adelaide, South Australia, Australia

${ }^{4}$ Discipline of Surgery, Adelaide Medical School, The University of Adelaide, Adelaide, South Australia, Australia

${ }^{5}$ Adelaide Medical School, The University of Adelaide, Adelaide, South Australia, Australia

\section{Twitter Jon Karnon @jonkarnon}

Acknowledgements We would like to thank those who participated in this study.

Contributors CTP contributed to the study concept and design; acquisition, analysis and interpretation of data; drafting and critical revision of the manuscript. $\mathrm{EH}, \mathrm{JK}, \mathrm{CLG}$ and RAF contributed substantially to the study concept and design; analysis and interpretation of data; critical revision for important intellectual content. All authors (CTP, CLG, RAF, JK, EH) approve of the final version to be published; and agree to be accountable for all aspects of the work in ensuring that questions related to the accuracy or integrity of any part of the work are appropriately investigated or resolved.

Funding This work was supported by funding from the National Health and Medical Research Council Project Grant (APP1025140).

Competing interests Dr Catherine Gibb is a consultant physician in the High Risk Clinic at the Royal Adelaide Hospital and Queen Elizabeth Hospital. The remaining authors declare no conflict of interest.

Patient consent for publication Not required. 
Ethics approval Ethics approval to conduct the study was granted by the South Australian Department of Health Human Research Ethics Committee (HREC Protocol No. 490/01/2015) and the Royal Adelaide Hospital Research Ethics Committee (RAH Approval No. 120225/120225a).

Provenance and peer review Not commissioned; externally peer reviewed.

Data availability statement № data are available.

Open access This is an open access article distributed in accordance with the Creative Commons Attribution Non Commercial (CC BY-NC 4.0) license, which permits others to distribute, remix, adapt, build upon this work non-commercially, and license their derivative works on different terms, provided the original work is properly cited, appropriate credit is given, any changes made indicated, and the use is non-commercial. See: http://creativecommons.org/licenses/by-nc/4.0/.

ORCID iD

Clarabelle T Pham http://orcid.org/0000-0003-4509-5368

\section{REFERENCES}

1 Kuwabara K, Imanaka Y, Matsuda S, et al. The association of the number of comorbidities and complications with length of stay, hospital mortality and Los high outlier, based on administrative data. Environ Health Prev Med 2008;13:130-7.

2 Librero J, Peiró S, Ordiñana R. Chronic comorbidity and outcomes of hospital care: length of stay, mortality, and readmission at 30 and 365 days. J Clin Epidemiol 1999;52:171-9.

3 Pham C, Gibb C, Field J, et al. Managing high-risk surgical patients: modifiable co-morbidities matter. ANZ J Surg 2014;84:925-31.

4 Pham CT, Gibb CL, Fitridge RA, et al. Effectiveness of preoperative medical consultations by internal medicine physicians: a systematic review. BMJ Open 2017;7:e018632.
5 Pham CT, Gibb CL, Mittinty MN, et al. A comparison of propensity score-based approaches to health service evaluation: a case study of a preoperative physician-led clinic for high-risk surgical patients. $J$ Eval Clin Pract 2016;22:761-70.

6 Klassen AC, Creswell J, Plano Clark VL, et al. Best practices in mixed methods for quality of life research. Qual Life Res 2012;21:377-80.

7 Braun V, Clarke V. Using thematic analysis in psychology. Qual Res Psychol 2006;3:77-101.

8 Thilen SR, Bryson CL, Reid RJ, et al. Patterns of preoperative consultation and surgical specialty in an integrated healthcare system. Anesthesiology 2013;118:1028-37.

9 Wijeysundera DN, Austin PC, Beattie WS, et al. Variation in the practice of preoperative medical consultation for major elective noncardiac surgery: a population-based study. Anesthesiology 2012;116:25-34.

10 Minick P, Harvey S. The early recognition of patient problems among medical-surgical nurses. Medsurg Nurs 2003;12:291-7.

11 Newell A, Simon HA. Human problem solving: Prentice-Hall Englewood cliffs, NJ, 1972.

12 Pearson $\mathrm{H}$. Science and intuition: do both have a place in clinical decision making? Br J Nurs 2013;22:212-5.

13 Crebbin W, Beasley SW, Watters DAK. Clinical decision making: how surgeons do it. ANZ J Surg 2013;83:422-8.

14 Standing M. Perceptions of clinical decision-making skills on a developmental journey from student to staff nurse. University of Kent, 2005.

15 Francis DMA. Surgical decision making. ANZ J Surg 2009;79:886-91.

16 Benner P, Tanner C. Clinical judgment: how expert nurses use intuition. Am J Nurs 1987;87:23-31.

17 Williams DB. The clinical history. Med J Aust 2017;207:473-5.

18 Lakdawalla DN, Doshi JA, Garrison LP, et al. Defining Elements of Value in Health Care-A Health Economics Approach: An ISPOR Special Task Force Report [3]. Value Health 2018;21:131-9.

19 Rosenstein AH. Physician communication and care management: the good, the bad and the ugly. Physician Exec 2012;38:34-7. 